\title{
Incidence and Relative Bio-Efficacy of Different Insecticides against Chaetoprocta (Chaetoprocta odata Hewitson) Infesting Walnut in Kashmir Valley
}

\author{
Arshad Abbas ${ }^{1}$, Nazir Ahmad Wani ${ }^{1}$, Sheikh Bilal Ahmad ${ }^{1}$, Abdul Rouf Wani ${ }^{1}, \&$ Mohammad Munib ${ }^{2}$ \\ ${ }^{1}$ Division of Entomology Sher-e-Kashmir University of Agricultural Sciences \& Technology of Kashmir \\ Shalimar Campus, Jammu \& Kashmir, India \\ ${ }^{2}$ Central Institute of Temperate Horticulture K. D. Farm, Old Air Field, Jammu \& Kashmir, India \\ Correspondence: Arshad Abbas, Division of Entomology Sher-e-Kashmir University of Agricultural Sciences \& \\ Technology of Kashmir Shalimar Campus, Jammu \& Kashmir 191 121, India. E-mail: \\ arshad.abbas5@gmail.com
}

Received: March 25, 2015 Accepted: July 20, 2015 Online Published: August 15, 2015

doi:10.5539/jas.v7n9p212 URL: http://dx.doi.org/10.5539/jas.v7n9p212

\begin{abstract}
The investigations entitled "Incidence and relative efficacy of different insecticides against Chaetoprocta (Chaetoprocta odata Hewitson) infesting walnut in Kashmir valley" were undertaken in three major walnut growing areas i.e., Salar (Anantnag), Wussan (Ganderbal) and Kulangam (Kupwara) districts of Kashmir Valley for two cropping seasons 2011 and 2012. Incidence of Chaetoprocta (Chaetoprocta odata Hewitson) revealed that the pest appeared in second fortnight of March on the terminal twigs and attained its peak population of 65.80 (2010) and 68.80 (2011) in the $1^{\text {st }}$ week of May at Salar (Anantnag). Pooled relative efficacy of different insecticides in field trials at three locations during 2010 and 2011 revealed that high mean per cent mortality was registered by imidacloprid $200 \mathrm{SL}$ at 0.03 per cent at all the three locations with pooled mean per cent mortality of (91.78) followed by dimethoate $30 \mathrm{EC}$ at 0.05 per cent (88.71).
\end{abstract}

Keywords: walnut, Chaetoprocta, incidence, management

\section{Introduction}

Walnut (Juglans regia Linnaeus) belongs to family Juglandaceae and genus Juglans is believed to have origin in Iran. In India, walnut production was earlier confined to Jammu \& Kashmir but now it is also cultivated in Himachal Pradesh, Uttarakhand hills. Besides India, major walnut growing countries are China, USA, Iran, Ukraine, Turkey and Mexico. China stands first followed by USA, Turkey and Iran while India stands seventh in production list. Walnut is an important crop grown in Jammu and Kashmir and contributes around 98 per cent of the country's production. The state produces about 86,263 tonnes from an area of 61,723 hectares. Walnuts are rich in amino acids and are involved in many functions of homeostatic regulation, protection against oxidative stress and has antioxidant potential (Canella \& Dernini 2004; Abbasi et al., 2010). India exported 9073.38 metric tonnes of walnut valued at Rs 1978.15 million during the period 2009-10 as against 5696.33 metric tonnes valued at Rs 1412.36 million in 2008-09 and is projected to reach around 75,000 tonnes by 2020 (Vigneshwara, 2012).

The insect pest Chaetoprocta (Chaetoprocta odata Hewitson) reported from walnut in Kashmir valley cause considerable damage to the crop as defoliators (Mir \& Wani, 2005). Chaetoprocta (Chaetoprocata odata Hewitson) is a monophagous pest having only one generation in a year, which starts its activity from March till July. Egg stage is the overwintering stage of the pest which lasts from July to March. Larval stage starts from the last week of March and extend to May having the $2^{\text {nd }}$ instar larvae as the most damaging stage. Pupation of the pest starts from the last week of May to June and emergence of adults take place in the months of June and July.

So far studies on different walnut pests have been carried out throughout the world but walnut pest Chaetoprocta (Chaetoprocta odata Hewitson) has remained unnoticed by and large though causing severe damage to walnut crop in Kashmir valley. Thus it was considered pertinent to undertake the proposed studies on management of Chaetoprocta (Chaetoprocta odata Hewitson) on walnut in Kashmir. 


\section{Materials and Methods}

\subsection{Sampling Procedure for Incidence}

Incidence and severity of infestation by leaf defoliators were ascertained at three representative sites viz., Anantnag, Ganderbal and Kupwara. Ten trees at each location were selected randomly which were not given insecticidal treatments.

The incidence was calculated as per formulae:

$$
\frac{\text { No of trees infested }}{\text { No of assessed trees }} \times 100
$$

From each tree 50 leaves were taken randomly from four geographical directions for recording severity of leaf infestation and sampling was done six times fortnightly during the pest activity period from March to June. The leaves having more than 50 per cent of area damage by $C$. odata were taken into account. The per cent leaf damage was calculated as follows:

$$
\frac{\text { Mean number of leaves damaged }}{\text { Total number of leaves marked }} \times 100
$$

\subsection{Management of Chaetoprocta odata}

For the purpose of evaluating the efficacy of different insecticides against Chaetoprocta odata caterpillars, healthy walnut plants of 15 years old were selected at every location of study. The experiment was laid out with the following details:

Design: Randomised Block Design;

Treatments for management: Chemicals $5+1$ control $=6$;

The pesticides used for management of Chaetoprocta odata were:

\section{Trade name}

Dursban 20EC

Rogor 30EC

Margocide 20EC

Farsa 10EC

Imida 200SL

\section{Active ingradient}

Chlorpyriphos

Dimethoate

Neem

Alphamethrin

Imidacloprid

\section{A.I Concentration (\%)}

0.05

0.05

0.03

0.05

0.03

Water (Control): water spray;

Replication: 3;

Unit of application: Tree as a whole.

\subsection{Method of Application}

Insecticidal suspension was prepared by mixing thoroughly the required quantity of the chemical in half a litre of clean tap water and then additional volume of water was added to make the required spray solution. The solution was agitated to make it homogenous and sprayed with a high pressure power sprayer and 20-25 litres spray solution per plant was sprayed. In case of check only water was sprayed. The spray was applied in the first week of May when damage is maximum and pest population of second instar larvae which causes maximum damage to the crop is at its peak at Salar (Anantnag) and Wussan (Ganderbal) but at Kulangam (Kupwara) it was applied in the second week during early morning hours of the day to avoid drift for two years of 2010 and 2011.

\subsection{Statistical Analysis}

The means of data, standard deviation, standard error of means, regression equations and other statistics were computed with the help of methods given by Box et al. (1978) using R-software provided by the Division of Agricultural Statistics, SKUAST-K, Shalimar Campus.

\section{Results}

3.1 Incidence of Chaetoprocta (Chaetoprocta odata Hewitson) at Salar (Anantnag), Wussan (Ganderbal) and Kulangam (Kupwara) during 2010 and 2011

The pooled mean data of two consecutive years of 2010 and 2011 at Salar (Anantnag) location indicated that pest population increased from 18.34 to 20.58 with a pooled mean pest load of $19.46 \pm 0.71$ at an average maximum and minimum temperature of $19.29 \pm 1.16$ and $5.85 \pm 0.27^{\circ} \mathrm{C}$, maximum and minimum relative humidity of 
$80.92 \pm 2.69$ and $50.81 \pm 3.68$ per cent, and rain fall of $3.44 \pm 0.09 \mathrm{~mm}$. Incidence on the basis of number of tress infested was cent per cent. It is evident from the correlation matrix computed for the year 2010 (Table 2a) that there existed positive correlations of $\mathrm{r}_{1}=0.41, \mathrm{r}_{2}=0.48$ between the population build up of the pest and the independent effect of maximum and minimum temperatures respectively. Strong positive correlation $\left(\mathrm{r}_{4}=0.85\right.$ and $\left.r_{5}=0.94\right)$ were worked out at $(0.05)$ and $(0.01)$ levels of significance with minimum relative humidity and mean rainfall, respectively. However, the effects of maximum relative humidity indicated negative correlation coefficients of $r_{3}=-0.41$ with the pest population build up. The same trend has been observed for the year 2011 . There was positive correlations of $r_{1}=0.39, r_{2}=0.20, r_{4}=0.88$ and $r_{5}=0.86$ between the population build up of the pest and the independent effect of maximum, minimum temperatures, at $(0.05)$ level of significance, minimum relative humidity and mean rainfall respectively. However, the effects of maximum relative humidity indicated negative correlation coefficients of $r_{3}=-0.11$ ) (Table 2b). The pooled mean data of two consecutive years of 2010 and 2011 at Wussan (Ganderbal) indicate that the pooled mean of pest population has increased from 6.93 to 7.38 with a pooled mean of $7.15 \pm 0.15$ at an average maximum and minimum temperature of 20.29 \pm 0.94 and $7.25 \pm 0.43{ }^{\circ} \mathrm{C}$, maximum and minimum relative humidity of $85.61 \pm 0.04$ and $54.39 \pm 1.35$ per cent, and rain fall of $2.35 \pm 0.42 \mathrm{~mm}$. Incidence on the basis of number of tress infested was 55 per cent (Table 1). It is evident from the correlation matrix computed for the year 2010 (Table 3a) that there existed positive correlations for $\left(r_{1}=0.42, r_{2}=0.49\right)$ between the population build up of the pest and the independent effect of maximum and minimum temperatures respectively. Strong positive correlations were worked out for minimum relative humidity and mean rainfall $\left(\mathrm{r}_{4}=0.89\right.$ and $\left.\mathrm{r}_{5}=0.86\right)$ at $(0.01)$ level of significance. However, the effects of maximum relative humidity indicated negative correlation coefficients $\left(\mathrm{r}_{3}=-0.28\right)$. But the trend changed during the year 2011. There existed positive correlations $\left(r_{1}=0.14, r_{2}=0.38, r_{5}=0.52\right)$ between the population build up of the pest and the independent effect of maximum temperature, minimum temperatures and mean rainfall, positive but non significant correlation was observed for minimum relative humidity $\left(r_{4}=0.78\right)$. However, the effects of Maximum relative humidity indicated negative correlation coefficients $\left(r_{3}=-0.14\right)$ (Table 3b). The mean data of two consecutive years of 2010 and 2011 at Kulangam (Kupwara) location indicate that the pooled mean of pest population has increased from 6.14 to 7.74 with a pooled mean of $6.94 \pm 0.56$ at maximum and minimum temperature of $23.25 \pm 0.64$ and $7.74 \pm 0.24{ }^{\circ} \mathrm{C}$, maximum and minimum relative humidity of $83.38 \pm$ 0.36 and $55.97 \pm 0.41$ per cent and rainfall of $3.06 \pm 0.06 \mathrm{~mm}$ respectively. Incidence on the basis of number of tress infested is 35 per cent (Table 1). It is evident from the correlation matrix computed for the year 2010 that there existed weak positive correlations for $\left(r_{1}=0.49, r_{2}=0.49, r_{3}=0.15\right)$ between the population build up of the pest and the independent effect of maximum, minimum temperatures and maximum relative humidity. However minimum relative humidity and mean rainfall $\left(\mathrm{r}_{4}=0.59\right.$ and $\left.\mathrm{r}_{5}=0.70\right)$ at $(0.01)$ level of significance showed a strong but non significant positive correlation (Table 4a). During the year 2011 their existed positive correlations for $\left(r_{1}=0.43, r_{2}=0.47, r_{3}=0.05, r_{4}=0.75\right)$ between the population build up of the pest and the independent effect of maximum temperature, minimum temperature, maximum and minimum relative humidity however, the effects of rain fall $\left(\mathrm{r}_{5}=0.93\right)$ indicated strong and significant positive correlation coefficients at $(0.01)$ level of significance (Table 4b). Metrological data was obtained from Agro meteorological Services of SKUAST-Kashmir Shalimar Campus. 
Table 1. Pooled mean on incidence of Chaetoprocta odata Hewitson in walnut orchards for 2010 \& 2011 at Salar (Anantnag), Wussan (Ganderbal) and Kulangam (Kupwara)

\begin{tabular}{|c|c|c|c|c|c|c|c|c|}
\hline & $\begin{array}{l}\text { Year of } \\
\text { observation }\end{array}$ & $\begin{array}{l}\text { *No. of tress } \\
\text { infested }\end{array}$ & $\begin{array}{l}* * \text { Pooled } \\
\text { mean of pest } \\
\text { population }\end{array}$ & $\begin{array}{l}\text { Mean of } \\
\text { max. Temp. } \\
\left({ }^{\circ} \mathrm{C}\right)\end{array}$ & $\begin{array}{l}\text { Mean of } \\
\text { min. Temp. } \\
\left({ }^{\circ} \mathrm{C}\right)\end{array}$ & $\begin{array}{l}\text { Mean of } \\
\text { max. RH } \\
(\%)\end{array}$ & $\begin{array}{l}\text { Mean of } \\
\text { min. RH } \\
(\%)\end{array}$ & $\begin{array}{l}\text { Mean of } \\
\text { rainfall } \\
(\mathrm{mm})\end{array}$ \\
\hline \multirow{3}{*}{$\begin{array}{l}\text { Salar } \\
\text { (Anantnag) }\end{array}$} & 2010 & 10 & 18.34 & 17.65 & 6.25 & 77.11 & 45.60 & 3.31 \\
\hline & 2011 & 10 & 20.58 & 20.94 & 5.46 & 84.74 & 56.02 & 3.57 \\
\hline & \multicolumn{2}{|c|}{ Pooled Mean \pm Sem } & $19.46 \pm 0.71$ & $19.29 \pm 1.16$ & $5.85 \pm 0.27$ & $80.92 \pm 2.69$ & $50.81 \pm 3.68$ & $3.44 \pm 0.09$ \\
\hline \multirow{3}{*}{$\begin{array}{l}\text { Wussan } \\
\text { (Ganderbal) }\end{array}$} & 2010 & 5 & 6.93 & 18.96 & 6.63 & 85.54 & 52.47 & 2.95 \\
\hline & 2011 & 6 & 7.38 & 21.62 & 7.87 & 85.68 & 56.31 & 1.75 \\
\hline & \multicolumn{2}{|c|}{ Pooled Mean \pm Sem } & $7.15 \pm 0.15$ & $20.29 \pm 0.94$ & $7.25 \pm 0.43$ & $85.61 \pm 0.04$ & $54.39 \pm 1.35$ & $2.35 \pm 0.42$ \\
\hline \multirow{3}{*}{$\begin{array}{l}\text { Kulangam } \\
\text { (Kupwara) }\end{array}$} & 2010 & 3 & 6.14 & 24.17 & 8.09 & 82.86 & 56.56 & 3.16 \\
\hline & 2011 & 4 & 7.74 & 22.34 & 7.39 & 83.90 & 55.38 & 2.97 \\
\hline & \multicolumn{2}{|c|}{ Pooled Mean \pm Sem } & $6.94 \pm 0.56$ & $23.25 \pm 0.64$ & $7.74 \pm 0.24$ & $83.38 \pm 0.36$ & $55.97 \pm 0.41$ & $3.06 \pm 0.06$ \\
\hline
\end{tabular}

Note. *: Incidence on the basis of Number of trees infested is 100, 55 and 35 per cent respectively; **: Data based on 10 replicates each.

Table 2a. Correlation matrix showing relationship between Chaetoprocta odata Hewitson infestation (Y) and abiotic factors $\left(\mathrm{X}_{1}, \mathrm{X}_{2}, \mathrm{X}_{3}, \mathrm{X}_{4}\right.$, and $\left.\mathrm{X}_{5}\right)$ at Salar (Anantnag) during 2010

\begin{tabular}{lllllll}
\hline & $\begin{array}{l}\text { Chaetoprocta odata } \\
\text { infestation }\end{array}$ & $\begin{array}{l}\text { Maximum } \\
\text { temperature }\end{array}$ & $\begin{array}{l}\text { Minimum } \\
\text { temperature }\end{array}$ & $\begin{array}{l}\text { Maximum relative } \\
\text { humidity }\end{array}$ & $\begin{array}{l}\text { Minimum relative } \\
\text { humidity }\end{array}$ & Rainfall \\
\hline $\mathrm{Y}$ & $\mathrm{X}_{1}$ & $\mathrm{X}_{2}$ & $\mathbf{X}_{3}$ & $\mathbf{X}_{4}$ & $\mathbf{X}_{5}$ \\
\hline $\mathrm{X}_{1}$ & $1.00^{* *}$ & 0.412 & 0.488 & -0.410 & $0.854^{*}$ & $0.948^{* *}$ \\
$\mathrm{X}_{2}$ & & & $0.879^{*}$ & $-0.824^{*}$ & 0.266 & 0.313 \\
$\mathrm{X}_{3}$ & & & $-0.988^{* *}$ & -0.236 & 0.504 \\
$\mathrm{X}_{4}$ & & & & -0.131 & -0.454 \\
$\mathrm{X}_{5}$ & & & & & $0.846^{*}$ \\
\hline Note. & & & & & $1.00^{* *}$ \\
\hline
\end{tabular}

Table 2b. Correlation matrix showing relationship between Chaetoprocta odata Hewitson infestation (Y) and abiotic factors $\left(\mathrm{X}_{1}, \mathrm{X}_{2}, \mathrm{X}_{3}, \mathrm{X}_{4}\right.$, and $\left.\mathrm{X}_{5}\right)$ at Salar (Anantnag) during 2011

\begin{tabular}{lllllll}
\hline $\begin{array}{l}\text { Chaetoprocta odata } \\
\text { infestation }\end{array}$ & $\begin{array}{l}\text { Maximum } \\
\text { temperature }\end{array}$ & $\begin{array}{l}\text { Minimum } \\
\text { temperature }\end{array}$ & $\begin{array}{l}\text { Maximum relative } \\
\text { humidity }\end{array}$ & $\begin{array}{l}\text { Minimum relative } \\
\text { humidity }\end{array}$ & Rainfall \\
\hline$Y$ & $\mathbf{X}_{1}$ & $\mathbf{X}_{2}$ & $\mathbf{X}_{3}$ & $\mathbf{X}_{4}$ & $\mathbf{X}_{5}$ \\
\hline$X_{1}$ & $1.00^{* *}$ & 0.398 & .204 & -0.118 & $0.882 *$ & $0.863^{*}$ \\
$X_{2}$ & & & $0.863^{*}$ & -0.548 & 0.678 & 0.521 \\
$X_{3}$ & & & -0.756 & -0.457 & 0.300 \\
$X_{4}$ & & & & & -0.315 & 0.256 \\
$X_{5}$ & & & & & & $0.962 * *$ \\
\hline
\end{tabular}

Note. * $(\mathrm{P}=0.05) ; *(\mathrm{P}=0.01)$. 
Table 3a. Correlation matrix showing relationship between Chaetoprocta odata Hewitson infestation (Y) and abiotic factors $\left(\mathrm{X}_{1}, \mathrm{X}_{2}, \mathrm{X}_{3}, \mathrm{X}_{4}\right.$, and $\left.\mathrm{X}_{5}\right)$ at Wussan (Ganderbal) during 2010

\begin{tabular}{lllllll}
\hline & $\begin{array}{l}\text { Chaetoprocta odata } \\
\text { infestation }\end{array}$ & $\begin{array}{l}\text { Maximum } \\
\text { temperature }\end{array}$ & $\begin{array}{l}\text { Minimum } \\
\text { temperature }\end{array}$ & $\begin{array}{l}\text { Maximum relative } \\
\text { humidity }\end{array}$ & $\begin{array}{l}\text { Minimum relative } \\
\text { humidity }\end{array}$ & Rainfall \\
\hline $\mathrm{Y}$ & $\mathrm{X}_{1}$ & $\mathrm{X}_{2}$ & $\mathrm{X}_{3}$ & $\mathrm{X}_{4}$ & $\mathrm{X}_{5}$ \\
$\mathrm{X}_{1}$ & & 0.428 & 0.496 & -0.287 & $0.896^{*}$ & $0.868^{*}$ \\
$\mathrm{X}_{2}$ & & & $0.915^{* *}$ & $-0.842^{*}$ & 0.662 & 0.502 \\
$\mathrm{X}_{3}$ & & & $-0.908^{* *}$ & -0.761 & 0.597 \\
$\mathrm{X}_{4}$ & & & & -0.648 & 0.397 \\
$\mathrm{X}_{5}$ & & & & & $0.895^{*}$ \\
\hline
\end{tabular}

Note. $*(\mathrm{P}=0.05) ; *(\mathrm{P}=0.01)$.

Table 3b. Correlation matrix showing relationship between Chaetoprocta odata Hewitson infestation (Y) and abiotic factors $\left(\mathrm{X}_{1}, \mathrm{X}_{2}, \mathrm{X}_{3}, \mathrm{X}_{4}\right.$, and $\left.\mathrm{X}_{5}\right)$ at Wussan (Ganderbal) during 2011

\begin{tabular}{lllllll}
\hline & $\begin{array}{l}\text { Chaetoprocta odata } \\
\text { infestation }\end{array}$ & $\begin{array}{l}\text { Maximum } \\
\text { temperature }\end{array}$ & $\begin{array}{l}\text { Minimum } \\
\text { temperature }\end{array}$ & $\begin{array}{l}\text { Maximum relative } \\
\text { humidity }\end{array}$ & $\begin{array}{l}\text { Minimum relative } \\
\text { humidity }\end{array}$ & Rainfall \\
\hline $\mathrm{Y}$ & $\mathrm{X}_{1}$ & $\mathrm{X}_{2}$ & $\mathrm{X}_{3}$ & $\mathrm{X}_{4}$ & $\mathrm{X}_{5}$ \\
\hline $\mathrm{Y}$ & $1.00^{* *}$ & 0.143 & 0.388 & -0.142 & 0.783 & 0.527 \\
$\mathrm{X}_{1}$ & & & $0.844^{*}$ & -0.761 & 0.517 & 0.077 \\
$\mathrm{X}_{2}$ & & & & $-0.882^{*}$ & -0.638 & 0.239 \\
$\mathrm{X}_{3}$ & & & & -0.498 & -0.048 \\
$\mathrm{X}_{4}$ & & & & & 0.149 \\
$\mathrm{X}_{5}$ & & & & & & $1.00^{* *}$ \\
\hline
\end{tabular}

Note. * $(\mathrm{P}=0.05) ; *(\mathrm{P}=0.01)$.

Table 4a. Correlation matrix showing relationship between Chaetoprocta odata Hewitson infestation (Y) and abiotic factors $\left(\mathrm{X}_{1}, \mathrm{X}_{2}, \mathrm{X}_{3}, \mathrm{X}_{4}\right.$, and $\left.\mathrm{X}_{5}\right)$ at KVK Kulangam (Kupwara) during 2010

\begin{tabular}{lllllll}
\hline & $\begin{array}{l}\text { Chaetoprocta odata } \\
\text { infestation }\end{array}$ & $\begin{array}{l}\text { Maximum } \\
\text { temperature }\end{array}$ & $\begin{array}{l}\text { Minimum } \\
\text { temperature }\end{array}$ & $\begin{array}{l}\text { Maximum relative } \\
\text { humidity }\end{array}$ & $\begin{array}{l}\text { Minimum relative } \\
\text { humidity }\end{array}$ & Rainfall \\
\hline $\mathrm{Y}$ & $\mathrm{X}_{1}$ & $\mathrm{X}_{2}$ & $\mathrm{X}_{3}$ & $\mathrm{X}_{4}$ & $\mathrm{X}_{5}$ \\
\hline $\mathrm{Y}$ & $1.00^{* *}$ & 0.495 & 0.496 & 0.153 & 0.598 & 0.706 \\
$\mathrm{X}_{1}$ & & & 0.763 & -0.380 & 0.376 & 0.634 \\
$\mathrm{X}_{2}$ & & & -0.726 & -0.501 & 0.769 \\
$\mathrm{X}_{3}$ & & & & -0.27 & -0.349 \\
$\mathrm{X}_{4}$ & & & & & $0.868^{*}$ \\
$\mathrm{X}_{5}$ & & & & & $1.00^{* *}$ \\
\hline Note.
\end{tabular}


Table 4b. Correlation matrix showing relationship between Chaetoprocta odata Hewitson infestation (Y) and abiotic factors $\left(\mathrm{X}_{1}, \mathrm{X}_{2}, \mathrm{X}_{3}, \mathrm{X}_{4}\right.$, and $\left.\mathrm{X}_{5}\right)$ at KVK Kulangam (Kupwara) during 2011

\begin{tabular}{lllllll}
\hline & $\begin{array}{l}\text { Chaetoprocta odata } \\
\text { infestation }\end{array}$ & $\begin{array}{l}\text { Maximum } \\
\text { temperature }\end{array}$ & $\begin{array}{l}\text { Minimum } \\
\text { temperature }\end{array}$ & $\begin{array}{l}\text { Maximum relative } \\
\text { humidity }\end{array}$ & $\begin{array}{l}\text { Minimum relative } \\
\text { humidity }\end{array}$ & Rainfall \\
\hline $\mathrm{Y}$ & $\mathrm{X}_{1}$ & $\mathrm{X}_{2}$ & $\mathrm{X}_{3}$ & $\mathrm{X}_{4}$ & $\mathrm{X}_{5}$ \\
$\mathrm{X}_{1}$ & $1.00^{* *}$ & 0.438 & 0.473 & 0.052 & 0.750 & $0.939^{* *}$ \\
$\mathrm{X}_{2}$ & & & $0.965^{* *}$ & -0.707 & 0.214 & 0.626 \\
$\mathrm{X}_{3}$ & & & -0.787 & 0.314 & 0.682 \\
$\mathrm{X}_{4}$ & & & & & 0.003 & -0.244 \\
$\mathrm{X}_{5}$ & & & & & & 0.744 \\
\hline
\end{tabular}

Note. $*(\mathrm{P}=0.05) ; *(\mathrm{P}=0.01)$.

3.2 Relative Bio-Efficacy of Different Insecticides against C. odata Hewitson at Salar (Anantnag), Wussan (Ganderbal) and Kulangam (Kupwara)

\subsubsection{Location 1}

At Salar (Anantnag) location during the year 2010, fifteen days after treatment (DAT) the per cent mortality was maximum with imidacloprid (93.95) followed by dimethoate (91.66), alphamethrin (81.86) and chlorpyriphos (75.62) however; it was minimum with the application of neem (60.28) (Table 5). Dimethoate and imidacloprid were statistically at par, however other treatments varied significantly. During the year 2011 the per cent mortality was persistently maximum with imidacloprid (93.02) followed by dimethoate (92.75) which were statistically at on par with each other. 80.50 and 74.69 per cent mortality was showed by alphamethrin and chlorpyriphos which were also statistically at par. However per cent mortality was minimum with the application of neem (56.80) which varied significantly with other treatments.

Table 5. Relative Bio-efficacy of different insecticides against $C$. odata Hewitson at Salar (Anantnag), Wussan (Ganderbal) and Kulangam (Kupwara) during cropping seasons of 2010 \& 2011

\begin{tabular}{|c|c|c|c|c|c|c|c|c|c|}
\hline \multirow[b]{2}{*}{ Treatments } & \multirow[b]{2}{*}{ Pesticide Used } & \multirow[b]{2}{*}{ Trade name } & \multirow[b]{2}{*}{ Concentration used } & \multicolumn{3}{|c|}{ Mean per cent mortality 2010} & \multicolumn{3}{|c|}{ Mean per cent mortality 2011} \\
\hline & & & & $\begin{array}{l}{ }^{*} \text { SITE } 1 \\
\text { 15DAT }\end{array}$ & $\begin{array}{l}\text { *SITE } 2 \\
\text { 15DAT }\end{array}$ & $\begin{array}{l}{ }^{*} \text { SITE } 3 \\
\text { 15DAT }\end{array}$ & $\begin{array}{l}{ }^{*} \text { SITE } 1 \\
\text { 15DAT }\end{array}$ & $\begin{array}{l}\text { *SITE } 2 \\
\text { 15DAT }\end{array}$ & $\begin{array}{c}{ }^{*} \text { SITE } 3 \\
\text { 15DAT }\end{array}$ \\
\hline T1 & Dimethoate 30 EC & Rogor & 0.05 & $\begin{array}{l}91.66 \mathrm{e} \\
(73.21)\end{array}$ & $\begin{array}{l}86.62 \mathrm{~d} \\
(68.54)\end{array}$ & $\begin{array}{l}80.07 \mathrm{e} \\
(63.48)\end{array}$ & $\begin{array}{l}92.75 \mathrm{~d} \\
(74.38)\end{array}$ & $\begin{array}{l}86.32 \mathrm{~d} \\
(68.29)\end{array}$ & $\begin{array}{l}77.80 \mathrm{~d} \\
(61.89)\end{array}$ \\
\hline $\mathbf{T 2}$ & Neem 20 EC & Margocide & 0.03 & $\begin{array}{l}60.28 \mathrm{~b} \\
(50.93)\end{array}$ & $\begin{array}{l}62.74 \mathrm{~b} \\
(52.38)\end{array}$ & $\begin{array}{l}47.21 \mathrm{~b} \\
(43.40)\end{array}$ & $\begin{array}{l}56.80 \mathrm{~b} \\
(48.90)\end{array}$ & $\begin{array}{l}68.73 \mathrm{~b} \\
(56.00)\end{array}$ & $\begin{array}{l}59.33 \mathrm{~b} \\
(50.33)\end{array}$ \\
\hline $\mathbf{T 3}$ & Chlorpyriphos 20 EC & Dursban & 0.05 & $\begin{array}{l}75.62 \mathrm{c} \\
(60.41)\end{array}$ & $\begin{array}{l}72.53 \mathrm{c} \\
(58.39)\end{array}$ & $\begin{array}{l}64.64 \mathrm{c} \\
(53.51)\end{array}$ & $\begin{array}{l}74.69 \mathrm{c} \\
(59.79)\end{array}$ & $\begin{array}{l}71.14 \mathrm{~b} \\
(57.50)\end{array}$ & $\begin{array}{l}66.22 \mathrm{c} \\
(54.46)\end{array}$ \\
\hline T4 & Alphamethrin 10EC & Farsa & 0.05 & $\begin{array}{l}81.86 \mathrm{~d} \\
(64.79)\end{array}$ & $\begin{array}{l}70.43 \mathrm{c} \\
(57.05)\end{array}$ & $\begin{array}{l}69.61 \mathrm{~d} \\
(56.54)\end{array}$ & $\begin{array}{l}80.50 \mathrm{c} \\
(63.79)\end{array}$ & $\begin{array}{l}75.84 \mathrm{c} \\
(60.55)\end{array}$ & $\begin{array}{l}68.51 \mathrm{c} \\
(55.86)\end{array}$ \\
\hline T5 & Imidacloprid 200SL & Imida & 0.03 & $\begin{array}{l}93.95 \mathrm{e} \\
(75.76)\end{array}$ & $\begin{array}{l}89.26 \mathrm{~d} \\
(70.87)\end{array}$ & $\begin{array}{l}84.15 \mathrm{e} \\
(66.53)\end{array}$ & $\begin{array}{l}93.02 \mathrm{~d} \\
(74.68)\end{array}$ & $\begin{array}{l}88.28 \mathrm{~d} \\
(69.98)\end{array}$ & $\begin{array}{l}86.20 \mathrm{e} \\
(68.19)\end{array}$ \\
\hline T6 & Water & & & $\begin{array}{l}1.82 \mathrm{a} \\
(7.75)\end{array}$ & $\begin{array}{l}0.03 \mathrm{a} \\
(0.99)\end{array}$ & $\begin{array}{l}0.07 \mathrm{a} \\
(1.50)\end{array}$ & $\begin{array}{l}1.52 \mathrm{a} \\
(7.08)\end{array}$ & $\begin{array}{l}0.32 \mathrm{a} \\
(3.24)\end{array}$ & $\begin{array}{l}0.27 \mathrm{a} \\
(2.95)\end{array}$ \\
\hline $\mathrm{CD}(\mathrm{p} \leq 0.05)$ & & & & 5.27 & 4.29 & 3.86 & 6.13 & 4.28 & 2.29 \\
\hline
\end{tabular}

Note. Data based on mean of three replications each; *Site 1, 2 and $3^{\text {rd }}$ stands for Salar (Anantnag), Wussan (Ganderbal) and Kulangam (Kupwara); DAT: Days after treatment; Values in parenthesis are Arcsine transformed values; The value in individual columns superscripted by similar letter(s) do not differ significantly. 


\subsubsection{Location 2}

Data revealed that at Wussan (Ganderbal) during the year 2010 fifteen days after treatment the per cent mortality was maximum with imidacloprid (89.26) followed by dimethoate (86.62) which were statistically at on par with each other. 72.53 and 70.43 per cent mortality was exhibited by chlorpyriphos and alphamethrin which were also statistically at par with each other. However, it was minimum with the application of neem (62.74) which varied significantly with other treatments. However during the year 2011 fifteen days after treatment the per cent mortality was consistently maximum with imidacloprid (88.28) followed by dimethoate (86.32); alphamethrin (75.84) and chlorpyriphos (71.14), however, it was minimum with the application of neem (68.73). Statistically alphamethrin varied significantly with other treatments but imidacloprid was at par with dimethoate and chlorpyriphos was at on par with neem (Table 5).

\subsubsection{Location 3}

Results depicted in (Table 5) show that at Kulangam (Kupwara) during the year 2010 fifteen days after treatment the per cent mortality was maximum with imidacloprid (84.15) followed by dimethoate (80.07). Least per cent mortality of was recorded with the application of neem (47.21). However 69.61 and 64.64 per cent mortality was recorded by alphamethrin and chlorpyriphos respectively. All the treatments varied significantly with each other except imidacloprid and dimethoate which were statistically at par. The same treatments were repeated for the next year of 2011 and almost similar results were obtained. The per cent mortality was consistently maximum with imidacloprid (86.20) followed by dimethoate (77.80), alphamethrin (68.51) and chlorpyriphos (66.22). However, it was minimum (59.33) with the application of neem. Statistically chlorpyriphos and alphamethrin were at par but rest of the treatments varied significantly with each other. The study indicated the order in efficacy of insecticides based on mortality of the pest as imidacloprid $>$ dimethoate $>$ alphamethrin $>$ chlorpyriphos $>$ neem.

\section{Discussion}

The present studies carried out during 2010-2011 revealed that $C$. odata started its activity in the second fortnight of March at Salar (Anantnag). It was observed that pest shows a gradual increase in the population in the first fortnight of April and peak of pest population was attained during the first week of May. The present investigation is in agreement with Massodi and Trali (1987) who reported that the slug like larvae of $C$. odata are greenish in colour and flattened in shape. This is also in close proximity with Mir and Wani (2005) who also reported that $C$. odata is potentially serious pest from April to June in Kashmir valley. The present studies are also in agreement with Arun (2009) who reported that adults of $C$. odata remains active during the month of May. Present findings are in line with the work conducted by Dar et al. (1977) who reported that insecticidal treatments with benzene hexachloride, and endosulfan suppress the population levels of $L$. obfuscata which is the lepidopteron pest of walnut. The present investigation is also in agreement with Webb et al. (1988) who reported that defoliation by gypsy moth, $L$. dispar was achieved by implantation or injection of the systemic insecticides viz., acephate or methmidophos. The present in vestigation falls in line with Mohinder et al. (2007) reported that among insecticides, chlorpyriphos ( 0.04 per cent), endosulfan ( 0.05 per cent) and deltamethrin $(0.0028$ per cent) resulted in the highest larval mortality of $L$. obfuscata.

\section{References}

Abbasi, M. A., Raza, A., Riaz, T., Shahzadi, T., Rehman, J. M., Shahwar, D, Siddiqui, S.Z., Chaudhary, A.R. Ahmad, N. (2010). Investigation on the volatile constituents of Juglans regia and their in vitro antioxidant potential. Pak. Acad. Sci., 47, 137-141.

Arun, P. S. (2009). Butterflies of Kedarnath Musk Deer Reserve, Garhwal Himalyas. Indian Journal of Threatened Taxa, l(1), 37-48. http://dx.doi.org/10.11609/joTT.o1873.37-48

Box, G. E. P., Hunter, W. G., \& Hunter, J. S. (1978). Statistics for Experimenters. New York John Wiley and Sons.

Cannella, C., \& Dernini, S. (2004). Walnut: Insights and Nutrition Value. Proceedings of $V^{\text {th }}$ International Symposium- Sorrento (Italy) November 9-13 (p. 549).

Dar, G. A., Sheikh, A. G., \& Ganjoo, B. L. (1977). Relative efficacy of some insecticides in suppressing Lymantria obfuscata walker on apple trees in Kashmir. Pesticides, 11(10), 27-29.

Masoodi, M. A., \& Trali, A. R. (1987). Record of Chaetoprocta odata Hewitson (Lycaenidae: Lepidoptera) on walnut trees in Kashmir. Indian Journal of Plant Protection, 15, 213. 
Mir, G. M., \& Wani, M. A. (2005). Severity of infestation and damage to walnut plantation by important insect pests in Kashmir. Indian Journal of Plant Protection, 33(2), 188-193.

Mohinder, S., Gupta, R., \& Gupta, P. R. (2007). Biology and control of Indian Gypsy moth, Lymantria obfuscata on apple in Himachal Pradesh. Indian Journal of Plant Protection, 35(1), 104-105.

Vigneshwara, V. (2012). Walnuts: Need to expand cultivation. Market Survey (pp. 13-15).

Webb, R. E., Peiffer, R., Fuester, R. W., Thorpe, K. W., Calabrese, L., \& McLaughlin, J. M. (1998). An evaluation of the residual activity of traditional, safe, and biological insecticides against the gypsy moth. Journal of Arboriculture, 24(5), 286-293.

\section{Copyrights}

Copyright for this article is retained by the author(s), with first publication rights granted to the journal.

This is an open-access article distributed under the terms and conditions of the Creative Commons Attribution license (http://creativecommons.org/licenses/by/3.0/). 